Branch"C2020.

Available online

\title{
ULTRASMALL BACTERIA AND PERIODONTITIS - THE NOVEL LINK
}

\author{
Dr. Balaji Ganesh S \\ Department of Periodontics, Saveetha Dental College and Hospital, \\ Saveetha Institute of Medical and Technical Sciences, \\ Saveetha University, Chennai, Tamil Nadu, India
}

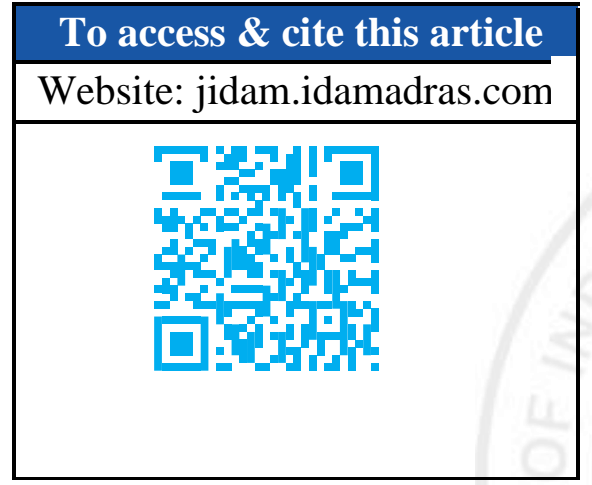

DOI:10.37841/jidam_2020_V7_I3_09

\section{Address for Correspondence:}

Dr. Balaji Ganesh. S., MDS., Senior lecturer, Department of Periodontics,

Saveetha Dental College and Hospital, Saveetha Institute of Medical and Technical Sciences, Saveetha University, Chennai 600077, Tamil Nadu, India Email id: balajiganeshs.sdc@ saveetha.com

$\begin{array}{ll}\text { Received } & : 30.08 .2020 \\ \text { Accepted } & : 09.09 .2020 \\ \text { Published } & : 27.09 .2020\end{array}$

\section{ABSTRACT}

Periodontal diseases are triggered by an interaction between bacterial components of tooth associated biofilms and host immune response mechanisms. Candidate phyla radiation (CPR) bacterial group is a recently identified, but highly diverse and abundant group of ultrasmall bacteria. CPR group includes Absconditabacteria (SR1), Gracilibacteria (GN02) and Saccharibacteria (TM7). Real time PCR and metagenomics based techniques are also employed to detect CPR species. TM7 organisms has been detected in periodontitis patients, HIVpositive patients with necrotizing ulcerative periodontitis, smokers with periodontitis, aggressive periodontitis cases and in mature plaque samples procured from orthodontic treatment undergoing individuals. It is found that TM7 isolate grow as short rods or cocci in dual-species biofilms with Porphyromonas gingivalis, Prevotella intermedia, Parvimonas micra or Streptococcus gordonii. The positive association of ultrasmall bacteria or CPR microorganisms with periodontal diseases spotlights the capacity of ultrasmall bacteria to modulate the immune response. The CPR microbiome is enriched with virulence factors and is adapted to a parasitic lifestyle that leads to disrupted host homeostasis. This makes the individual susceptible to periodontal tissue destruction. In future, we should instigate researches to know more about the actions and properties of ultrasmall bacteria and their association with periodontal diseases.

KEYWORDS: ultrasmall bacteria, periodontitis, Candidate Phyla Radiation (CPR) group, periodontal microbiology 


\section{INTRODUCTION:}

Periodontal diseases are inflammatory and destructive in nature; it affects the supporting tissues of teeth and ultimately leads to tooth loss if untreated and neglected. It is triggered by an interaction between bacterial components of tooth associated biofilms and host immune response mechanisms. Although it is multifactorial, specific gram-negative microorganisms in the subgingival plaque biofilm play a prime role in the initiation and progression of periodontitis. Red complex group of pathogens such as Porphyromonas gingivalis, Treponema denticola and Tannerella forsythia form an alliance in the subgingival biofilm and are considered as the prime periodontopathogenic bacteria $^{1}$. Bacteria exert pathogenic effects on the host directly by causing connective tissue destruction through various virulence factors or indirectly by triggering and modulating the host responses. Viruses such as herpes, cytomegalo, epstein barr and their association in the pathogenesis of periodontitis has already been explored in various studies. Viral infections generally diminishes the periodontal defenses, thereby allowing the subgingival overgrowth of various putative periodontopathic bacteria. Oral cavity also harbours certain ultrasmall bacteria which belongs to the newly identified and classified candidate phyla radiation (CPR) group ${ }^{2}$. This short communication deals with the association between ultrasmall bacteria and periodontitis.

\section{CANDIDATE PHYLA RADIATION (CPR) GROUP:}

CPR comprises of a group of ultrasmall bacteria with about $15 \%$ of all bacterial diversity. CPR consists of over 70 different phyla and two superphyla (Parcubacteria and Microgenomates). They are recently reported expansion of the tree of life with broad phylogenetic variation. CPR members have certain characteristics which includes inadequate biosynthetic capabilities such as they lack the ability to produce lipids, amino acids, certain conserved genes ${ }^{3}$. They are obligate fermenters with the complete absence of respiratory genes. But, members of the parcubacteria with new genomes were found to be involved in hydroxylamine oxidation and nitrate reduction to ammonia. CPR group includes Absconditabacteria (SR1), Gracilibacteria (GN02) and Saccharibacteria (TM7) ${ }^{4}$.

\section{DETECTION OF CPR GROUP BACTERIA:}

CPR microorganisms are little difficult to cultivate and detect with PCR based methods because of divergent genetic sequences. 16S rRNA gene-based identification is not sufficient for the classification of CPR microorganisms because of the divergence of CPR 16S rRNA sequences and the inadequacy of PCR primers commonly used for gene amplification. However, real time PCR has been used in some studies to detect CPR ${ }^{5}$. Metagenomics is a shotgun sequencing-based DNA sequencing methodology in which DNA isolated directly from the environment is sequenced and the reconstructed genome fragments are assigned to draft genomes. Metagenomics based techniques are also employed to detect CPR species ${ }^{6}$.

\section{EVIDENCES LINKING CPR WITH PERIODONTITIS:}

A number of studies have reported about the CPR microorganisms and their potential pathogenic associations in multiple mucosal diseases including inflammatory bowel disease, periodontitis and halitosis. A study which involved sequencing of the $16 \mathrm{~S}$ rRNA gene from 15 subgingival plaque samples from both healthy and periodontitis individuals concluded that TM7 organism were found to be $1 \%$ in healthy and it increased to $21 \%$ in periodontitis patients ${ }^{7}$. Furthermore, diseased samples shared a common structure that was not found in completely healthy samples, suggesting that the disease state may occupy a narrow region within the space of possible configurations of the oral microbiome. The analysis of the TM7 and Actinomyces genomes showed recombination events possibly linked with virulence factors ${ }^{8}$. Lateral transfer of virulence determinants occurs through phages. This leads to a partial separation between function and phylogeny thus, suggesting the need for metagenomic and functional analysis as a complement to taxonomic surveys of host associated microbiota. Thus, whole metagenome sequencing techniques helps in characterizing the genomes of prime players in the oral and periodontal microbiome. In another study, sequence and checkerboard analysis of subgingival plaque samples from $8 \mathrm{HIV}$-positive patients with necrotizing ulcerative periodontitis was done. They concluded that one of the most commonly detected organisms were those belonging to the phylum TM79. 
Subgingival plaque samples from 82 patients which included non-smoking periodontally healthy control group, non-smokers with periodontitis and smokers with periodontitis was analyzed in a study through $16 \mathrm{~S}$ pyrosequencing, in which TM7 was detected in both smokers and nonsmokers with periodontitis ${ }^{10}$. Another study investigated the presence of TM7 bacteria by real time quantitative PCR in human subgingival plaque samples obtained from healthy volunteers and periodontitis cases which was divided into 3 groups as mild, moderate and severe. TM7 rDNA was identified in $96 \%$ of the samples. TM7 bacteria was detected higher in mild periodontitis sites when compared to healthy and severe periodontitis sites ${ }^{11}$. In a pilot study,

18 patients who were previously diagnosed and underwent treatment for aggressive periodontitis were selected. Plaque samples were collected from healthy periodontal sites, residual periodontal pocket sites, healthy implant sites, perimucositis sites and periimplantitis sites. Out of which, TM7 phylum organisms was detected only in residual periodontal pocket sites ${ }^{12}$. TM7 organism have a parasitic lifestyle marked by the long term stable infection of bacterial hosts ${ }^{13}$. Candidate division TM7 bacterial phylotypes are also found in mature plaque samples procured from orthodontic bands of orthodontic treatment undergoing individuals. It is found that TM7 isolate grow as short rods or cocci in dual-species biofilms with Porphyromonas gingivalis, Prevotella intermedia, Parvimonas micra or Streptococcus gordonii. This clearly shows that TM7 organisms has the capacity to modulate biofilm growth of other oral bacteria to form biofilms with a range of different oral bacterial species and to be itself growth modulated by these species ${ }^{4}$. This interaction could enhance the development of a disease associated oral microbial community, thereby leading to the inflammatory and destructive changes of gingiva and periodontium.

\section{CONCLUSION:}

The positive association of ultrasmall bacteria or CPR microorganisms with periodontal diseases spotlights the capacity of ultrasmall bacteria to modulate the immune response. The CPR microbiome is enriched with virulence factors and is adapted to a parasitic lifestyle that leads to disrupted host homeostasis. This makes the individual susceptible to periodontal tissue destruction. The future is exciting for researchers in the field of periodontal diagnostics with the unraveling of the actions and properties of novel periodontal pathogens like ultrasmall bacteria.

\section{FINANCIALSUPPORTANDSPONSORSHIP:}

\section{Nil}

\section{CONFLICTS OF INTEREST:}

There are no conflicts of interest.

\section{REFERENCES:}

1. Page RC and Kornman KS. The pathogenesis of human periodontitis: An introduction. Periodontol 2000 1997; 14: 9-11.

2. Hug LA, Baker BJ, Anantharaman K, Brown CT, Probst AJ, Castelle CJ et al. A new view of the tree of life. Nat Microbiol 2016;1:16048.

3. Attar N. Bacterial evolution: CPR breathes new air into the tree of life. Nat Rev Microbiol 2016; 14(6):332-3.

4. Bor B, Bedree JK, Shi W, McLean JS, He X. Saccharibacteria (TM7) in the Human Oral Microbiome. J Dent Res 2019;98(5):500-509.

5. Soro V, Dutton LC, Sprague SV, Nobbs AH, Ireland AJ, Sandy JR et al. Axenic culture of a candidate division TM7 bacterium from the human oral cavity and biofilm interactions with other oral bacteria. Appl Environ Microbiol 2014;80(20):6480-6489.

6. Bikel S, Valdez-Lara A, Cornejo-Granados F, Rico K, Canizales-Quinteros S, Soberón, $\mathrm{X}$ et al. Combining metagenomics, metatranscriptomics and viromics to explore novel microbial interactions: towards a systems-level understanding of human microbiome. Comput Struct Biotechnol J 2015;13:390-401.

7. Liu B, Faller LL, Klitgord N, Mazumdar V, Ghodsi M, Sommer DD et al. Deep sequencing of the oral microbiome reveals signatures of periodontal disease. PLoS One 2012;7(6):e37919.

8. He X, McLean JS, Edlund A, Yooseph S, Hall AP, Liu SY et al. Cultivation of a humanassociated TM7 phylotype reveals a reduced genome and epibiotic parasitic lifestyle. Proc Natl Acad Sci U S A 2015;112(1):244-249. 
9. Paster BJ, Russell MK, Alpagot T, Lee AM, Boches SK, Galvin JL et al. Bacterial diversity in necrotizing ulcerative periodontitis in HIV-positive subjects. Ann Periodontol 2002;7(1):8-16.

10. Camelo-Castillo AJ, Mira A, Pico A, Nibali L, Henderson B, Donos N et al. Subgingival microbiota in health compared to periodontitis and the influence of smoking. Front Microbiol 2015;6:119.

11. Brinig MM, Lepp PW, Ouverney CC,Armitage GC, Relman DA. Prevalence of bacteria of division TM7 in human subgingival plaque and their association with disease. Appl Environ Microbiol 2003;69(3):1687-1694.

12. Sousa V, Nibali L, Spratt D, Dopico J, Mardas N, Petrie A et al. Peri-implant and periodontal microbiome diversity in aggressive periodontitis patients: a pilot study. Clin Oral Implants Res 2017;28(5):558-570.

13. Bor B, McLean JS, Foster KR, Cen L, To TT, Serrato-Guillen A et al. Rapid evolution of decreased host susceptibility drives a stable relationship between ultrasmall parasite TM7x and its bacterial host. Proc Natl Acad Sci U S A 2018;115(48):12277-12282. 